\title{
Increasing the Operating Speed of a Consequent Pole Axial Gap Motor for Higher Output Power Density
}

\author{
Toru Ogawa ${ }^{* a)}$ \\ Member, \\ Tomohira Takahashi* \\ Member \\ Masatsugu Takemoto** Member, \\ Satoshi Ogasawara** \\ Fellow \\ Hideaki Arita* \\ Member, \\ Akihiro Daikoku* \\ Member
}

(Manuscript received Aug. 21, 2018, revised Dec. 13, 2018)

\begin{abstract}
In this study, we examine how to increase the operating speed of a consequent pole axial gap motor to achieve higher output density. Our research group has been developing a consequent pole axial gap motor with field windings as the traction motor for electric vehicles. A smaller and lighter traction motor is required for better fuel economy and layout of such vehicles, and it is profitable to manufacture a smaller motor with an increased operating speed. To achieve high-speed operation, it is necessary to suppress the line-to-line voltage, and to that end, we examine the slot/pole combination. Moreover, because the rotor outer diameter of an axial gap motor is larger than that of a radial gap motor, the rotor strength should also be considered. We study the use of non-magnetic high-tensile-strength steel for the component that supports the rotor. We present the motor design and the results of spin burst tests, and we confirm that the motor can operate at the target maximum speed and output power density.
\end{abstract}

Keywords: motor, consequent pole, ferrite permanent magnet, axial gap, variable flux

\section{Introduction}

The regulations against vehicles with internal combustion engines are becoming stricter. Electric vehicles (EVs) are gaining attention as one solution to reduce $\mathrm{CO}_{2}$ emissions, and many car and component manufacturers are developing traction motors ${ }^{(1)-(3)}$. There are many necessary attributes for traction motors for EVs, for example variable flux capability and a flat shape; the latter is needed regarding the layout of the motor in the EV. Another necessary attribute is the ability to achieve simultaneously (i) high torque at low speed, (ii) high efficiency at low load, and (iii) a wide constant output. Generally, high magnetic flux is necessary for high torque, but if the magnetic flux in a motor is too large it is difficult to reduce the iron loss at low load. Moreover, high induced voltage when operating at high speed makes it difficult to achieve constant output over a wide speed range. Consequently, there is a trade-off relation between high torque and high efficiency or wide constant output characteristics.

In the study for variable flux capability, many types of motor have been developed ${ }^{(4)-(7)}$. To achieve variable flux capability and a flat shape simultaneously, our research group has proposed and has been developing an axial gap motor of the consequent-pole type with a ferrite permanent magnet (PM) with field windings ${ }^{(8)-(11)}$, overmore higher output

\footnotetext{
a) Correspondence to: Toru Ogawa. E-mail: Ogawa.Toru@dr. MitsubishiElectric.co.jp

* Mitsubishi Electric Corporation

8-1-1, Tsukaguchi-Honmachi, Amagasaki, Hyogo 661-8661, Japan

** Hokkaido University

Kita 14, Nishi 9, Kita-ku, Sapporo, Hokkaido 060-0814, Japan
}

density is also needed. Many research groups are investigating motors that operate at higher speed to increase the output density ${ }^{(12)(13)}$. Regarding the axial gap motor that we are proposing, it is profitable to increase the outer diameter of the rotor and decrease the motor length. Therefore, the radius and centrifugal force of an axial gap motor are generally larger than those of a radial-gap motor. This makes it necessary to attend to the larger centrifugal force when seeking to increase the operating speed of an axial gap motor. It is also necessary to suppress the line-to-line voltage to increase the operating speed.

Herein, we examine a design that increases the operating speed, and we show by three-dimensional (3D) finite-element analysis (FEA) that this design achieves the target output power density. To facilitate operation at maximum speed, we also examine the choice of material and structure for the rotor. Through burst tests on the rotor, we confirm its ability to operate at maximum speed. We assemble a proto sample and present test results.

\section{Proposed Motor}

2.1 Structure Figure 1 shows the proposed motor schematically, comprising an internal rotor and external stators. The rotor comprises PMs and rotor cores (SMCs). The SMCs are made of magnetic material and form the consequent poles. All the PMs are magnetized axially in the same direction. The PMs and SMCs are arranged circumferentially and alternately on the supporting component, which is made of non-magnetic material. There is not a back yoke on the rotor, and the magnetic flux passes through the rotor only axially. There is a stator on either side of the rotor with concentrated windings on each tooth. We choose concentrated 


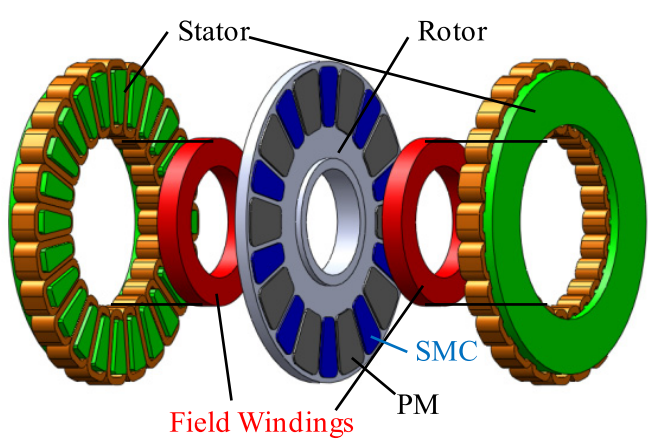

Fig. 1. Outline of proposed motor

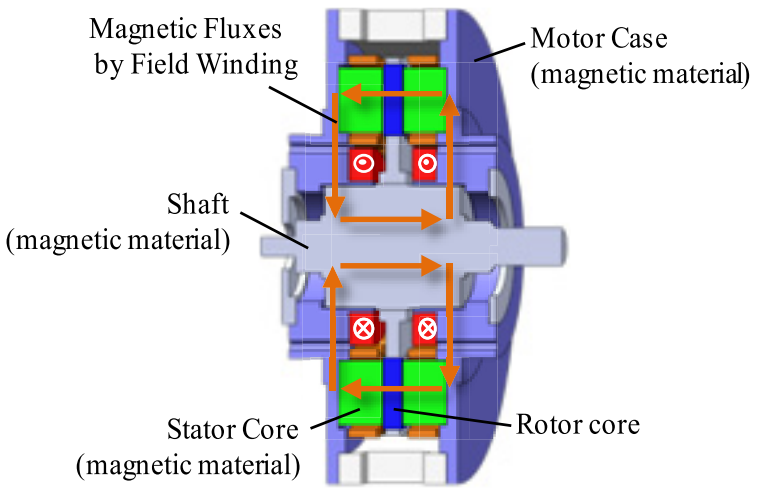

Fig. 2. Magnetic flux due to field winding current

windings so as not to increase the stator outer diameter, and we place field windings at the inner space of stator cores to utilize the dead space.

2.2 Variable Flux Capability We explain here how the proposed motor achieves variable flux capability. Figure 2 shows the proposed motor in cross section. A direct current (DC) is applied to each field winding to generate a magnetic flux; the DC currents are in the same direction. In Fig. 2, the arrows indicate the path of the magnetic flux generated by the DC winding current (also known as the field current). The magnetic flux due to the field current goes around the magnetic path that is formed by the motor shaft, motor case, stator core, rotor, stator core, and motor case on the other side.

Next, we show how the magnetic flux in the motor is altered. Figure 3 shows how the magnetic flux due to the field current goes through around the rotor. When no field current is applied (Fig. 3(a)), the magnetic flux comes from the PMs only and goes around the magnetic circuit formed by the teeth and back yoke of the stator core and the SMCs, teeth, and back yoke of the other stator. Consequently, magnetic poles appear on the SMCs with a direction that is opposite to that of the PMs. When a field current is applied in a direction chosen to generate a magnetic flux that opposes that of the PMs (Fig. 3(b)), that of the SMCs is strengthened. Thus, the magnetic flux density in the air gap (a fundamental component in the present case) increases. We refer to the direction of this field current as the strengthening direction. By contrast, if the field-current direction is chosen to generate a magnetic flux in the same direction as that of the PMs (Fig. 3(c)), the magnetic flux of the SMCs is weakened, thereby reducing the magnetic flux density; we refer to that direction as the

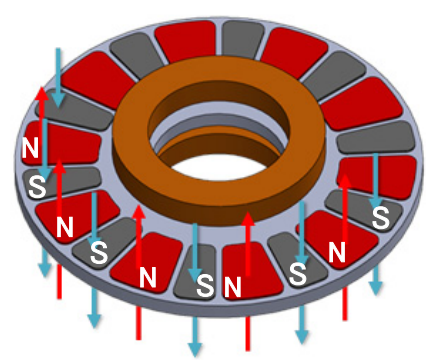

(a) Without field winding current

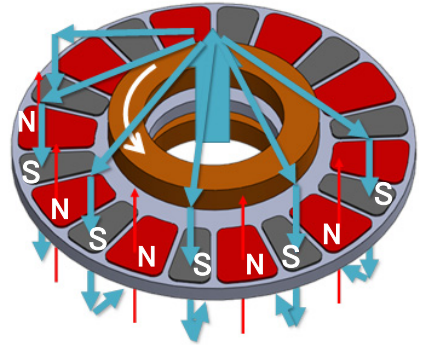

(b) With field-strengthening current

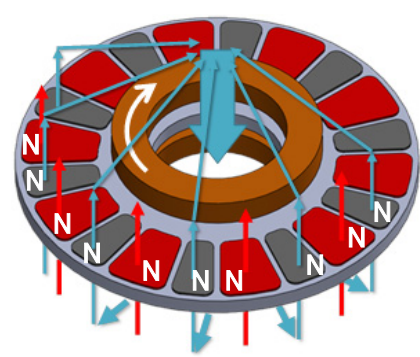

(c) With field-weakening current

Fig. 3. The path of magnetic flux due to the field current around the rotor

weakening direction. Therefore, as explained, the proposed motor has variable magnetic flux capability.

\section{Examination for Higher Output Density}

3.1 Slot/pole Combination We studied to increase output power density by 1.3 times compared to first type motor from $3.7 \mathrm{~kW} / \mathrm{L}$ to $4.8 \mathrm{~kW} / \mathrm{L}$. Table 1 compares the targeted design conditions between a motor of the first type ${ }^{(10)(11)}$ and one of the new design. The target output density of the latter is $4.8 \mathrm{~kW} / \mathrm{L}$. This higher output density is achieved by increasing the operating speed from 5,000 rpm to $12,000 \mathrm{rpm}$. The armature and field current densities of the new motor are

Table 1. Targeted properties of studied motors

\begin{tabular}{|c|c|c|}
\hline $\begin{array}{c}\text { Maximum armature } \\
\text { current }\end{array}$ & First type & New design \\
\hline $\begin{array}{c}\text { Maximum armature } \\
\text { current density }\end{array}$ & $11.9 \mathrm{~A}_{\mathrm{rms}} / \mathrm{mm}^{2}$ & $11.9 \mathrm{~A}_{\mathrm{rms}} / \mathrm{mm}^{2}$ \\
\hline $\begin{array}{c}\text { Maximum field } \\
\text { current }\end{array}$ & $5.5 \mathrm{~A}$ & $4.3 \mathrm{~A}$ \\
\hline $\begin{array}{c}\text { Maximum field } \\
\text { current density }\end{array}$ & $6.95 \mathrm{~A} / \mathrm{mm}^{2}$ & $5.5 \mathrm{~A} / \mathrm{mm}^{2}$ \\
\hline Base speed & $1,600 \mathrm{rpm}$ & $3,360 \mathrm{rpm}$ \\
\hline Maximum speed & $5,000 \mathrm{rpm}$ & $12,000 \mathrm{rpm}$ \\
\hline Output power density & $3.7 \mathrm{~kW} / \mathrm{L}$ & $4.8 \mathrm{~kW} / \mathrm{L}$ \\
\hline
\end{tabular}


Table 2. Winding factors of studied motors

\begin{tabular}{|c|c|c|c|c|}
\hline Pole number & 10 & \multicolumn{3}{|c|}{14} \\
\hline Slot number & 12 & 12 & 15 & 18 \\
\hline Fundamental & 0.933 & 0.933 & 0.951 & 0.902 \\
\hline 2nd order & 0 & 0 & 0.100 & 0 \\
\hline 4th order & 0 & 0 & 0.100 & 0 \\
\hline 5th order & 0.067 & 0.067 & 0.173 & 0.038 \\
\hline 7th order & 0.067 & 0.067 & 0.111 & 0.136 \\
\hline 8th order & 0 & 0 & 0.165 & 0 \\
\hline 10th order & 0 & 0 & 0.100 & 0 \\
\hline 11th order & 0.933 & 0.171 & 0.044 & 0.136 \\
\hline 13th order & 0.933 & 0.210 & 0.021 & 0.038 \\
\hline
\end{tabular}

either the same as or less than those of the motor of the first type. To increase operating speed of hybrid excitation consequent pole axial gap motor and achieve higher output power density, it is necessary to take following points into consideration, (i) suppress operating frequency as high as $1.5 \mathrm{kHz}$, (ii) suppress iron loss which increases under high frequency operation and keep high efficiency, (iii) make slot number and pole number as high as possible to reduce magnetic saturation in iron core, and (iv) suppress the influence of even-order harmonic components by selecting appropriate slot/pole combination. We choose 24 slots and 20 poles for the motor of the first type. With 20 poles, it is difficult to suppress the operating frequency under $1.5 \mathrm{kHz}$ when the maximum speed is increased to $12,000 \mathrm{rpm}$. Therefore, to suppress the operating frequency, it is necessary to have fewer poles. Our group has shown that the slot/pole combination of 12 slots and 10 poles is suitable for a motor with a consequent-pole rotor ${ }^{(9)(10)}$. Because the winding factors of even harmonic orders are zero, the influence of an asymmetrically distributed air-gap magnetic flux density can be canceled. However, because it is difficult to miniaturize a 10-pole motor we investigate changing the number of poles from 20 to 14 .

Table 2 lists the winding factors of a 14-pole motor along with those of a 12-slot/10-pole motor. Although the fundamental winding factor of the 15-slot/14-pole motor is the largest, the winding factors of even orders are not zero, and the even-order harmonic component of the rotor's magnetomotive force cannot be canceled. The even-order winding factors of the 12-slot and 18-slot motors are zero, making 12 or 18 slots suitable for the 14-pole motor. Comparing the magnetic fluxes between 12-slot motor and 18-slot motor, that of the 12-slot motor is larger. Optimizing the thickness of the back yoke for the 18-slot motor makes the magnetic flux density at the back yoke of the 12-slot motor excessively large, causing magnetic flux to leak to the cover, which is made of magnetic material. However, the cover is not made of laminated material, and very large iron loss occurs when operating at high speed. Consequently, we choose the 18-slot motor, which is suitable for miniaturization.

3.2 Rotor Strength Next, we consider the outer diameter of the rotor. Figure 4 shows the rotor structure of the proposed motor. The PMs and SMCs are glued to the rotor's supporting component, which is made of non-magnetic material. To avoid closing the circuit around the PMs and rotor cores, there are slits in the outer part of the rotor's supporting component to decrease the eddy current loss that is generated

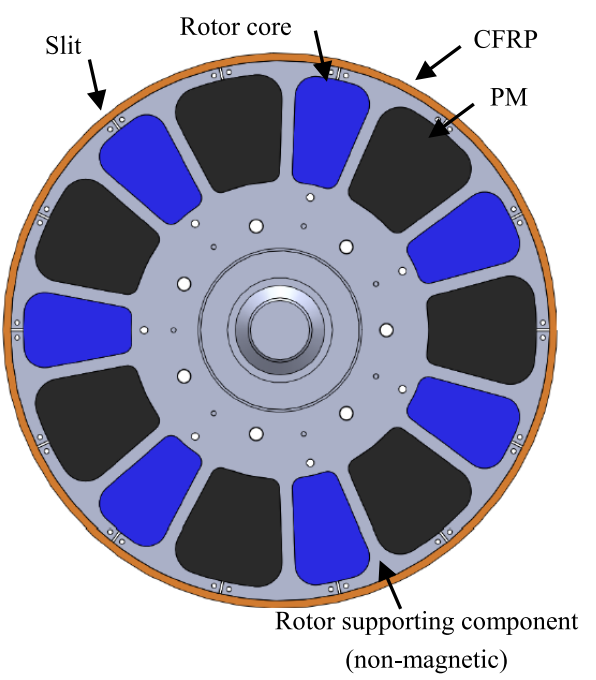

Fig. 4. Top view of rotor

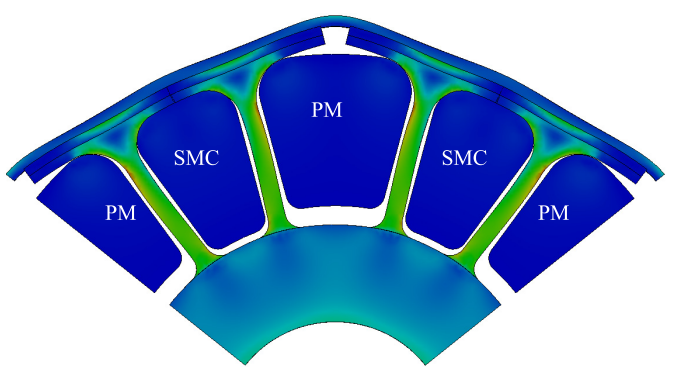

Fig. 5. Rotor displacement at maximum rotation speed (new design)

therein. The rotor's supporting component is reinforced with 3-mm-thick carbon fiber reinforced polymer (CFRP). We use SUS304 for the rotor's supporting component of the motor of the first type, the von Mises stress of which is $109.2 \mathrm{MPa}$ at the maximum speed of 5,000 rpm; we use a yield stress of $206 \mathrm{MPa}$ for SUS304. The von Mises stress is proportional to the square of the rotation speed and would exceed $600 \mathrm{MPa}$ at $12,000 \mathrm{rpm}$, making it clearly impossible to drive the motor up to $12,000 \mathrm{rpm}$ without countermeasures. Because the von Mises stress is generally proportional to the outer diameter of the rotor, we reduce the outer diameter of from $250 \mathrm{~mm}$ to $210 \mathrm{~mm}$.

We also consider the material of the rotor's supporting component. The Japan Steel Works produces a special material that is a non-magnetic high-tensile steel to which $18 \%$ manganese and $18 \%$ chromium is added $(18 \% \mathrm{Mn} 18 \% \mathrm{Cr}$ steel $)^{(14)}$. The material was developed for the retaining ring of an electric generator that required high yield strength; the yield stress of the material is 1,260 MPa. Figure 5 shows the distribution of rotor displacement at the maximum operating speed. The displacement is emphasized by 30 times. As PMs are designed to be wider than SMCs referring to the former examination $^{(11)}$, the weight of PMs are heavier than that of SMCs. Centrifugal force on PMs are greater than that on SMCs. And slit at the PMs widened. The von Mises stress at the maximum operating speed is $586.1 \mathrm{MPa}$, which is less than the yield stress of the material.

Figure 6 shows the rotor in cross section. The rotor's supporting component comprises three plates that are joined 


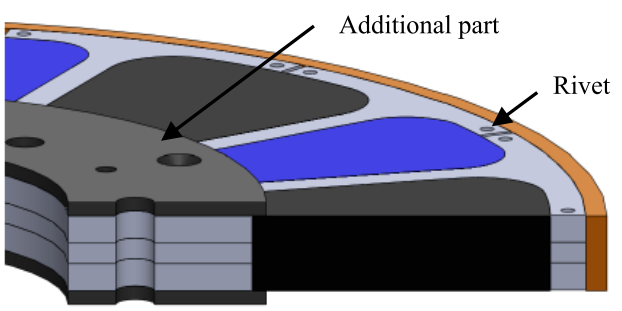

Fig. 6. Cross-sectional view of rotor

Table 3. Specifications of studied motors

\begin{tabular}{|c|c|c|}
\hline & First type & New design \\
\hline Number of poles & 20 & 14 \\
\hline Number of slots & 24 & 18 \\
\hline $\begin{array}{c}\text { Outer diameter } \\
\text { (coil end included) }\end{array}$ & $250 \mathrm{~mm}$ & $210 \mathrm{~mm}$ \\
\hline $\begin{array}{c}\text { Total motor } \\
\text { axial length }\end{array}$ & $76.4 \mathrm{~mm}$ & $77.8 \mathrm{~mm}$ \\
\hline Air gap & $1.0 \mathrm{~mm}$ & $1.0 \mathrm{~mm}$ \\
\hline
\end{tabular}

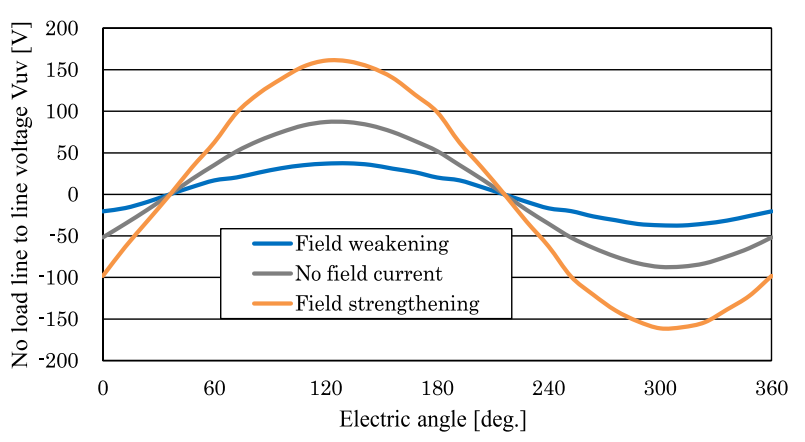

(a) Waveforms

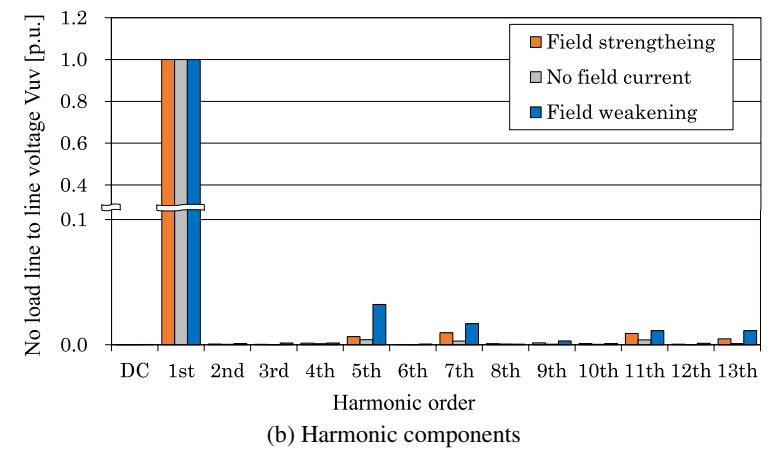

Fig. 7. No-load induced line-to-line voltage

using rivets. A part is added to the inner area of the rotor to prevent the PMs and SMCs from lifting under high-speed operation.

3.3 Motor Characteristics Table 3 compares the motor specifications of the motors of the first type and the new design. We confirm the motor characteristics by means of 3D FEA. Figure 7(a) shows waveforms of the no-load induced line-to-line voltage calculated with (i) the maximum field-weakening current, (ii) the maximum fieldstrengthening current, and (iii) no field current, and Fig. 7(b) shows the harmonic components. Figure 8 shows the noload induced line-to-line voltage versus the field winding current. The no-load induced line-to-line voltage is increased by $85.3 \%$ with the maximum field-strengthening current compared to that with no field winding current, and it is decreased

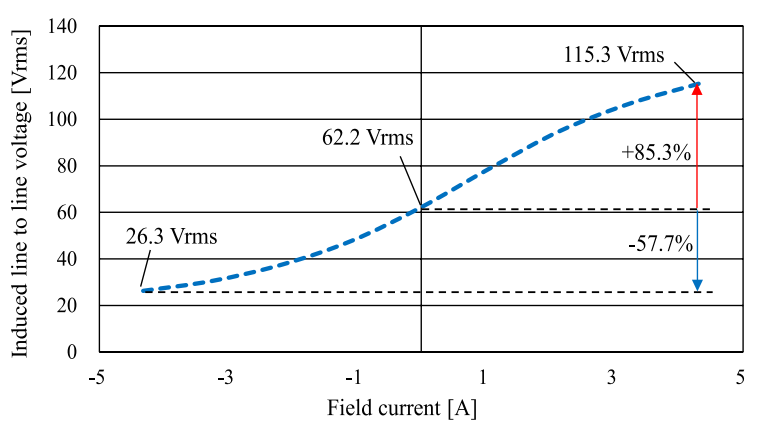

Fig. 8. No-load line-to-line voltage vs. field current

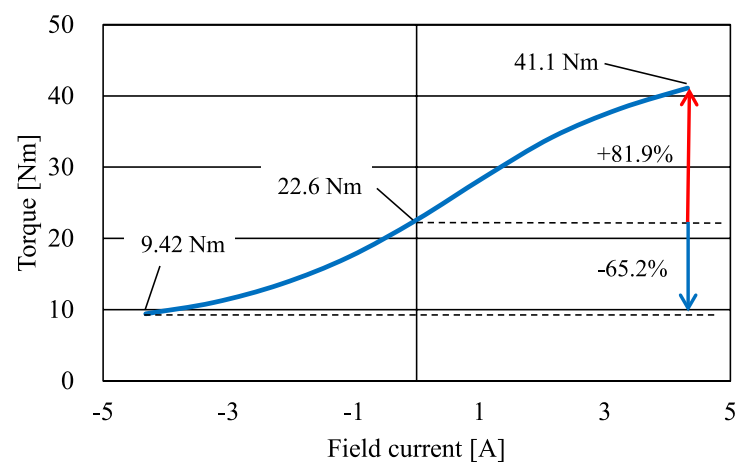

Fig. 9. Field-current-torque characteristic

by $57.7 \%$ with the maximum field-weakening current. Figure 9 shows the calculated output torque upon changing the field current from the maximum field-weakening current to the maximum field-strengthening current. The armature current is set to a maximum value of $74.8 \mathrm{~A}_{\mathrm{rms}}$. When no field current is applied, the average torque is $22.6 \mathrm{Nm}$. By applying the maximum field-strengthening current of $+4.3 \mathrm{~A}$, the torque is increased by $81.9 \%$ compared to that with no field current. The torque ripple is $11.9 \%$ under the maximum field-strengthening condition, whereas the torque with the maximum field-weakening current is reduced by $65.2 \%$. As expected, these results indicate that the magnetic flux can be controlled over a wide range by changing the field current.

When the motor is driven under high speed, eddy current loss should be considered. In the case of such motor as PMs and SMCs are supported by non-magnetic steel rotor supporting component, eddy current loss occurs in it. It is confirmed that eddy current loss could be suppressed by making slits at outer area of the rotor supporting component in (15). Eddy current losses with and without slits are compared under $3360 \mathrm{r} / \mathrm{min}$. Figure 10 shows eddy current loss density of rotor support component with and without slits. Without adding slits, eddy current loss of rotor supporting component around SMC is extremely large. By adding slits at outer area, eddy current loss can suppressed. Table 4 shows 3D-FEA results of eddy current losses and Eddy current loss of proposing motor can be reduced with adding slits at outer area of rotor supporting component from $1450 \mathrm{~W}$ to $36.8 \mathrm{~W}$. It is confirmed that there is an effect with the proposing motor to suppress eddy current loss by adding slits. Figure 11 shows the motor torque-speed characteristic. The torque at the base speed of 3,360 rpm is $41.7 \mathrm{Nm}$, and the output power is $14.7 \mathrm{~kW}$. The output power density is $5.4 \mathrm{~kW} / \mathrm{L}$, meaning that the newly designed motor can achieve higher than 


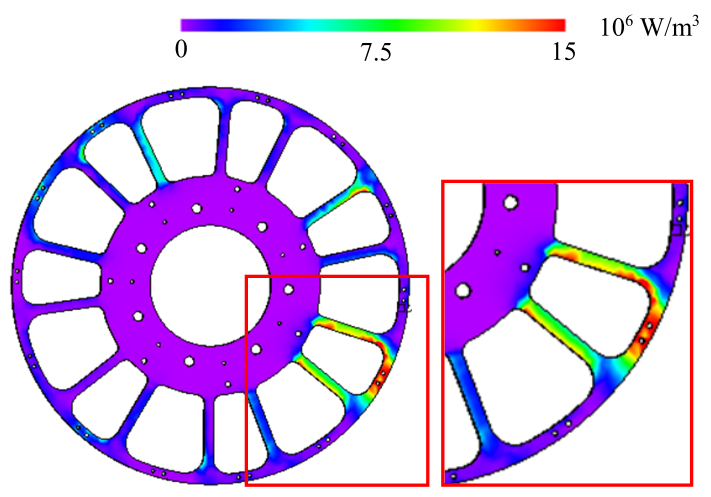

(a) Without slits

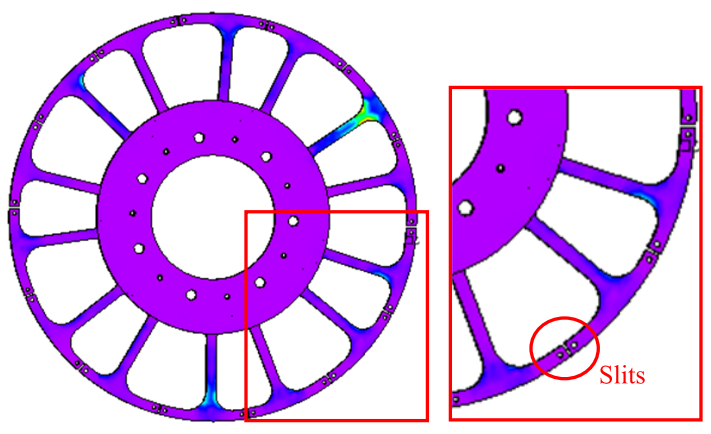

(b) With slits

Fig. 10. No-load induced line-to-line voltage

Table 4. Eddy current loss at rotor supporting component

\begin{tabular}{|c|c|}
\hline Without slits & With slit \\
\hline $1450.0 \mathrm{~W}$ & $36.8 \mathrm{~W}$ \\
\hline
\end{tabular}

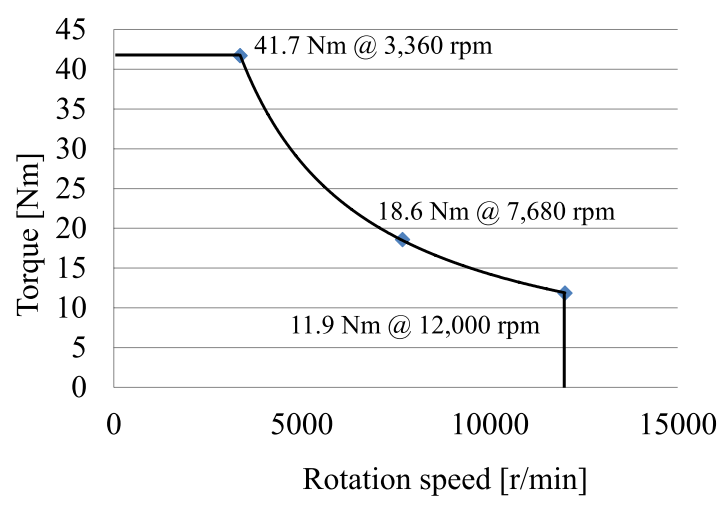

Fig. 11. Speed-torque characteristic

1.4 times output power density compared to first type motor and achieve the target output power density. The torque at the maximum speed of $12,000 \mathrm{rpm}$ is $11.9 \mathrm{Nm}$, and the output power is $14.9 \mathrm{~kW}$. It is confirmed that the motor can be driven at constant output power over a wide range of rotation speed from $3,360 \mathrm{rpm}$ to $12,000 \mathrm{rpm}$. It is also confirmed that the proposed motor can achieve higher output density by increasing its operating speed by using non-magnetic hightensile stainless steel for the rotor's supporting component and CFRP for reinforcement.

\section{Experimental Results}

4.1 High-speed Burst Tests To confirm the strength of the rotor, we performed high-speed burst tests. For these,

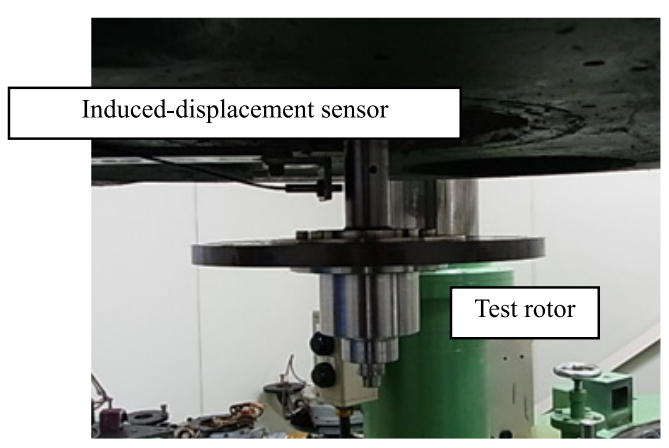

Fig. 12. Overview of high-speed burst tests

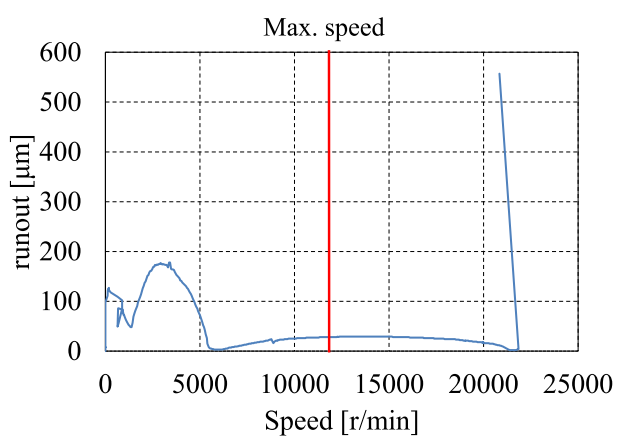

Fig. 13. Shaft runout versus rotation speed

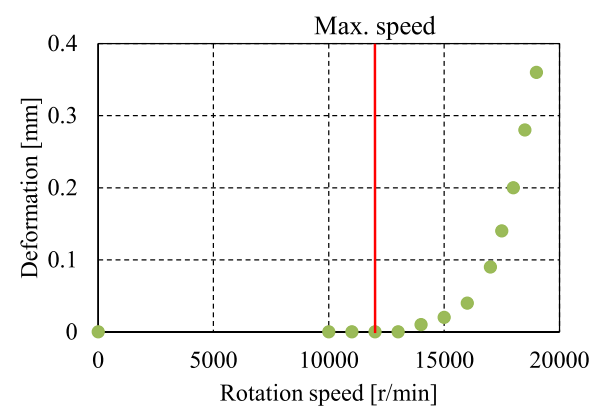

(a) Without CFRP

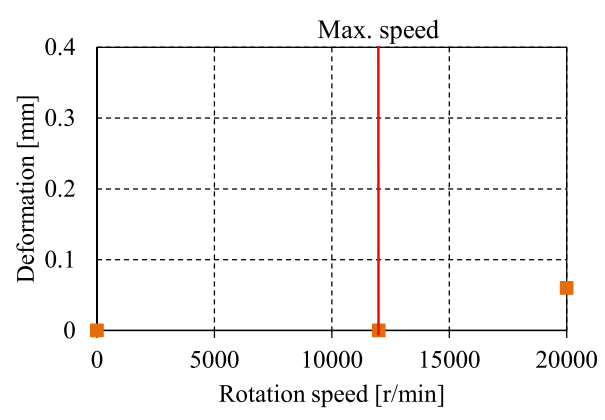

(b) With CFRP

Fig. 14. Rotor deformation with rotation speed

we used parts made of SUS304 instead of the actual PMs and SMCs because the specific gravities are almost the same. Figure 12 shows an overview of the high-speed burst tests. The shaft runout is measured with an induced-displacement sensor near the fixing point. Figure 13 shows the shaft runout versus the rotation speed. The tests were performed without CFRP. The rotation speed at which the runout increases rapidly $(\sim 21,800 \mathrm{rpm})$ is that at which the rotor disintegrated.

Figure 14 shows how the rotor's supporting component deformed with rotation speed. We measured the outer diameter 


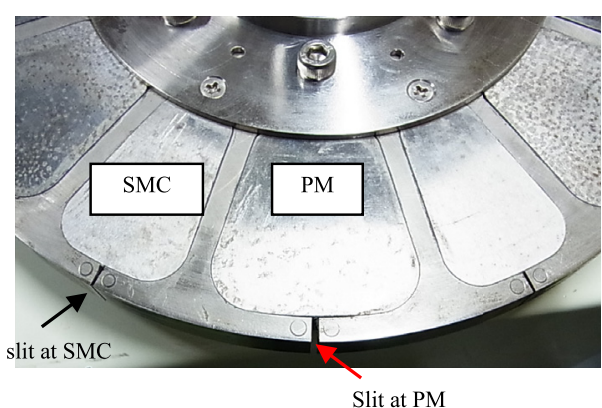

Fig. 15. Deformation of rotor's supporting component near slits

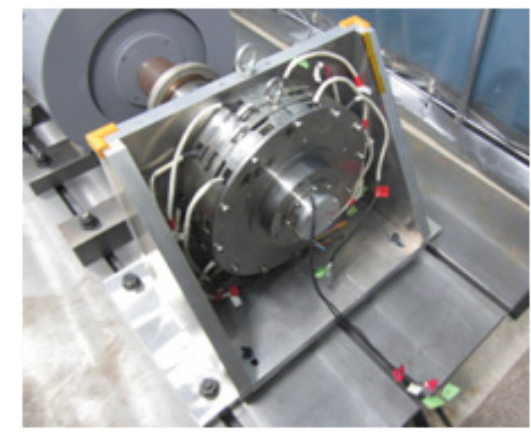

Fig. 16. Photograph of proto machine

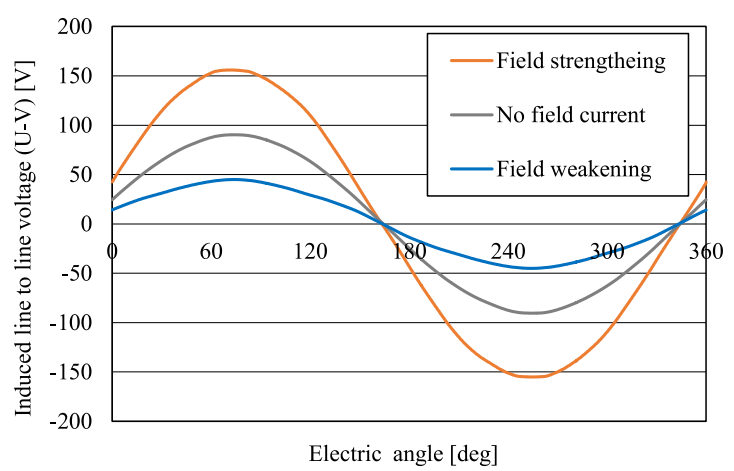

Fig. 17. Waveforms of no-load line-to-line voltage

after increasing the rotation speed using caliper. Figure 14(a) shows the results measured without CFRP. Even without CFRP, no irreversible deformation occurred at the maximum operating speed of $12,000 \mathrm{rpm}$, but irreversible deformation did occur once the speed exceeded 14,000 rpm. Figure 14(b) shows the deformation measured with CFRP; adding CFRP reduced the rotor deformation to a quarter of that without CFRP. Figure 15 shows a test rotor without CFRP after it had been rotated at 19,000 rpm. Because the PMs were heavier than the SMCs, the centrifugal force on the former was greater than that on the latter. Consequently, the slits at the PMs widened and those at the SMCs narrowed.

4.2 Test of Motor Characteristics Figure 16 shows a photograph of a proto machine that was assembled based on the specifications given in Table 3. Figure 17 shows waveforms of the no-load line-to-line voltage measured with (i) the maximum field-strengthening current, (ii) the maximum field-weakening current, and (iii) with no field winding current. The waveform distortion is small and the waveforms are close to sine waves.

Figure 18 shows the no-load induced line-to-line voltage

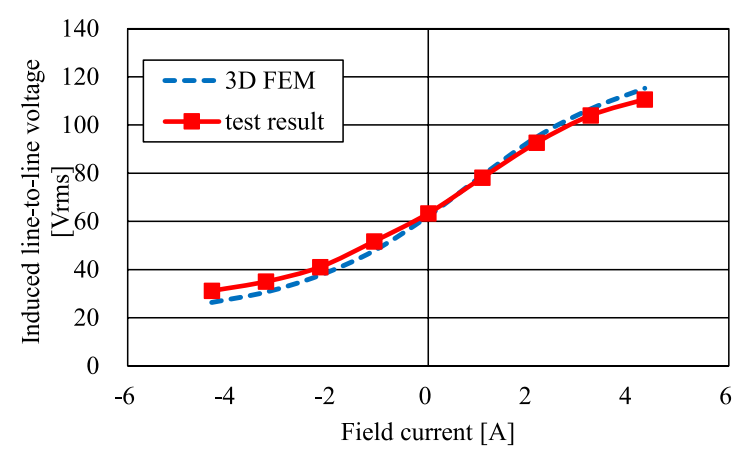

Fig. 18. No-load line-to-line voltage vs. field winding current

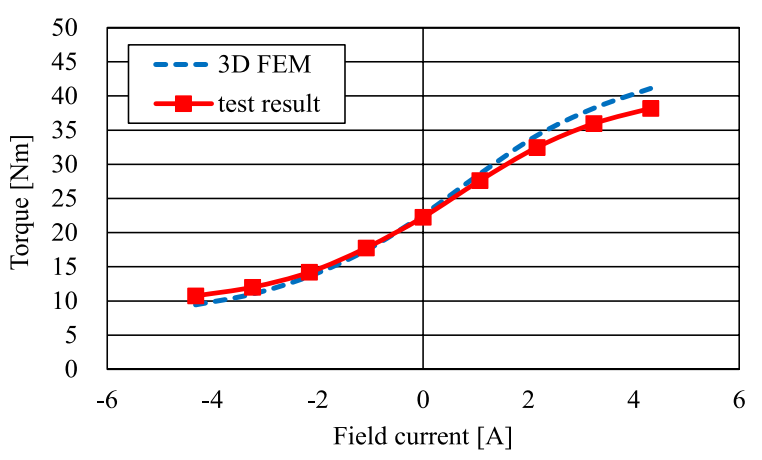

Fig. 19. Torque vs. field winding current

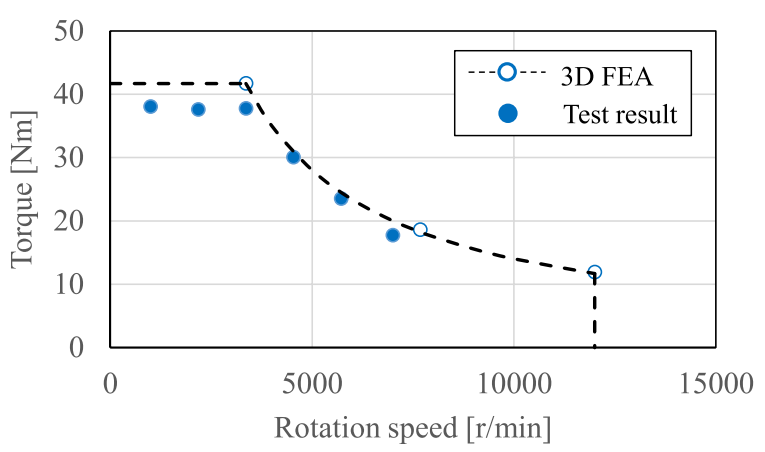

Fig. 20. Measured speed-torque characteristic

versus the field current measured at a base speed of 3,360 rpm; FEA results are also plotted. The test results coincide well with the FEA results. The induced line-toline voltage is increased by $74.7 \%$ with the maximum fieldstrengthening current and decreased by $50.8 \%$ with the maximum field-weakening current. The total harmonic distortions of the no-load line-to-line induced voltage with the maximum field-strengthening field current, with no field current, and with the maximum field-weakening current are $0.9 \%, 0.5 \%$, and $2.5 \%$, respectively. This test indicates that the proposed motor has variable flux capability by controlling the field winding current. Figure 19 shows torque versus the field winding current measured at the base speed. Torque also indicates the same trend as no load induced line-to-line voltage. Figure 20 shows measured speed-torque characteristic. Test was operated up to $7000 \mathrm{r} / \mathrm{min}$ due to ability of test bench. It is confirmed that under rotation speed of higher than the base speed, 3,360 r/min, motor output power is more than $13.3 \mathrm{~kW}$, which output power density $(4.9 \mathrm{~kW} / \mathrm{L})$ is larger by more than 1.3 times compared to first type motor $(3.7 \mathrm{~kW} / \mathrm{L})$, 
although test results are smaller than 3D-FEA results due to magnetic saturation in iron core. The target, 1.3 times larger output power density, can be achieved. It is considered that slot pole combination of 18 slots and 14 poles is suitable for hybrid excitation consequent pole axial gap motor to increase operation speed.

\section{Conclusion}

In this paper, we have presented an examination of increasing the operating speed of a consequent pole axial gap motor to achieve higher output density. For the countermeasure against centrifugal force of axial gap motor, a non-magnetic high-tensile stainless steel is applied as the rotor's supporting component and it is confirmed that the proposed motor could be operated at $12,000 \mathrm{rpm}$ by means of burst tests of the rotor. We designed the proposed motor using 3D FEA to achieve the target output power density higher than first type motor by 1.3 times. By selecting slot combination of 18 slots and 14 poles, operating frequency can be suppressed less than $1.5 \mathrm{kHz}$ at the maximum speed of $12,000 \mathrm{r} / \mathrm{min}$ and the influence of even-order harmonic due to the asymmetric air gap magnetic flux density distribution can be canceled with test of proto sample. A wide variable magnetic flux capability is also confirmed. Moreover, output power under higher than the base speed is more than $13.3 \mathrm{~kW}$ and output power density is $4.9 \mathrm{~kW} / \mathrm{L}$, which is more than the target, higher than first type motor by 1.3 times. It is considered that the slotpole combination of 18 slots and 14 poles is profitable for motor whose maximum speed is high and the combination of 24 slots and 20 poles is suitable for motor whose maximum speed is low or middle.

\section{References}

( 1 ) F. Momen, K. Rahman, Y. Son, and P. Savagian: "Electrical Propulsion System design of Chevrolet Bolt battery electric vehicle", ECCE 2016, pp.1-8 (2016)

( 2 ) T. Kato, N. Limsuwan, C.-Y. Yu, K. Akatsu, and R.D. Lorenz: "Rare Earth Reduction Using a Novel Variable Magnetomotive Force Flux-Intensified IPM Machine", IEEE Trans. on IAS, Vol.50, No.3, pp.1748-756 (2014)

( 3 ) M.M. Swamy, T. Kume, and A. Maemura: "Extended High-Speed Operation via Electronic Winding-Change Method for AC Motors", IEEE Trans. on Industry Applications, Vol.42, No.3, pp.742-752 (2006)

( 4 ) I. Ozawa, T. Kosaka, and N. Matsui: "Less Rare-Earth Magnet-High Power Density Hybrid Excitation Motor Designed for Hybrid Electric Vehicle Drives", Proc. European Conf. on Power Electronics and Appl., Barcelona, No.772 (2009)

( 5 ) H. Hijikata, K. Akatsu, Y. Miyama, H. Arita, and A. Daikoku: "Matrix Motor with Individual Winding Current Control Capability for Variable Parameters and Iron Loss Suppression", in Proc. of XXIst International Conference on Electrical Machine (ICEM) 2014, pp.546-552 (2014)

( 6 ) J.A. Tapia, F. Leonardi, and T. Lipo: "Consequent-Pole Permanent-Magnet Machine With Extended Field-Weakening Capability", IEEE Trans. on Industry Applications, Vol.39, No.6, pp.1704-1709 (2003)

( 7 ) F.G. Capponi, G.D. Donato, G. Borocci, and F. Caricchi: "Axial-Flux Hybrid-Excitation Synchronous Machine: Analysis, Design, and Experimental Evaluation", IEEE Trans. on Industry Applications, Vol.50, No.5, pp.3173-3184 (2014)

( 8 ) T. Takahashi, M. Takemoto, O. Satoshi, T. Ogawa, H. Arita, and A. Daikoku: "Operation Characteristics of a Consequent Pole PM type Axial-Gap Motor with DC Field Winding", The Papers of Joint Technical Meeting on "Motor Drive", "Rotating Machinery", "Vehicle Technology" IEE Japan, MD-15088, RM-15-069, VT-15-016, pp.81-86 (2015)

( 9 ) T. Ogawa, H. Arita, M. Nakano, A. Daikoku, T. Takahashi, M. Takemoto, and S. Ogasawara: "The Examination of Pole Number Combination of Consequent Pole Type Ferrite PM Axial Gap Motor with Field Winding", The
Papers of Technical Meeting on "Rotating Machinery" IEE Japan, RM-15163, pp.35-40 (2015)

(10) T. Ogawa, T. Takahashi, M. Takemoto, H. Arita, A. Daikoku, and S. Ogasawara: "The Consequent-Pole Type Ferrite Magnet Axial Gap Motor with Field Winding for traction motor used in EV", EVTeC \& APE 2016, p.6 (2016)

(11) T. Ogawa, T. Takahashi, M. Takemoto, S. Ogasawara, and A. Daikoku: "The Examination of Pole Geometry of Consequent Pole Type Ferrite PM Axial Gap Motor with Field Winding", IEMDC 2017 (2017)

(12) T. Yashiro, S. Sano, K. Takizawa, and T. Mizutani: "Development of New Motor for Compact-Class Hybrid Vehicle", EVTeC \& APE 2016, p.6 (2016)

(13) K. Ueta and K. Akatsu: "Study of high-speed SRM with Amorphous steel sheet for EV", ICEMS 2016 (2016)

(14) K. Orita, Y. Ikeda, T. Iwadate, and J. Ishizaka: "Development and Production of $18 \mathrm{Mn}-18 \mathrm{Cr}$ Non-magnetic Retaining Ring with High Yield Strength”, ISIJ International, Vol.30, No.8, pp.587-593 (1990)

(15) K. Sone, M. Takemoto, S. Ogasawara, K. Takezaki, and W. Hino: "Examination for the Higher Efficiency in a Ferrite Permanent Magnet $10 \mathrm{~kW}$ In-Wheel Axial-Gap Motor with Coreless Rotor Structure", Proc. of ECCE 2014, pp.5885-5892 (2014)

Toru Ogawa (Member) was born in Kanagawa, Japan, in 1980. He

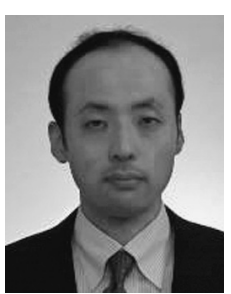
received B.S. and M.S. degrees in electrical engineering from Tokyo University, Japan, in 2003 and 2005, respectively. In 2013, he joined the Advanced Technology R\&D Center of the Mitsubishi Electric Corporation. He is engaged in research on DC motors, permanent-magnet synchronous motors, and axialgap motors, which are used for automobiles.

Tomohira Takahashi (Member) was born in Sapporo, Japan, in

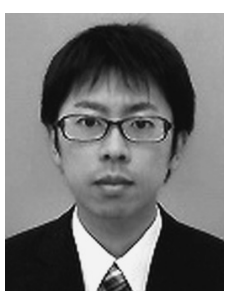
1991. He received B.S. and M.S. degrees from the Graduate School of Information Science and Technology, Hokkaido University, Japan, in 2014 and 2016, respectively. He is engaged in research on axial-gap motors using ferrite permanent magnets. Mr. Takahashi is a member of IEEE and the Institute of Electrical Engineers of Japan (IEEJ).

Masatsugu Takemoto (Member) was born in Tokyo, Japan, in 1972.

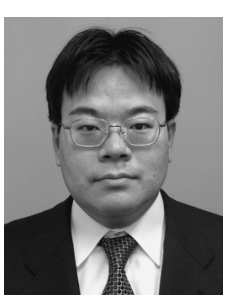
He received B.S. and M.S. degrees in electrical engineering from Tokyo University of Science, Japan, in 1997 and 1999, respectively, and a Ph.D. degree in electrical engineering from the Tokyo Institute of Technology, Japan, in 2005. In 1999, he joined the Tokyo Institute of Technology as a Research Associate in the Department of Electrical Engineering. In 2004, he joined the Musashi Institute of Technology, Tokyo, as a Research Associate in the Department of Mechanical Systems Engineering, where he became a Lecturer in 2005. Since 2008, he has been with Hokkaido University, Japan, where he is an Associate Professor in the Division of Systems Science and Informatics. He is engaged in research on permanent-magnet synchronous motors, axial gap motors, rare-earth-free motors, bearingless motors, and magnetic bearings. 
Satoshi Ogasawara (Fellow) was born in Kagawa, Japan, in 1958. He

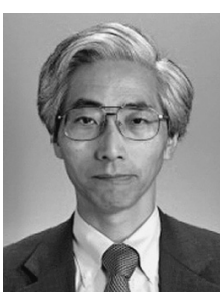
received B.S., M.S., and Dr.Eng. degrees in electrical engineering from the Nagaoka University of Technology, Japan, in 1981, 1983, and 1990, respectively. From 1983 to 1992, he was a Research Associate at Nagaoka University of Technology. From 1992 to 2003, he has with the Department of Electrical Engineering, Okayama University, Japan. From 2003 to 2007, he was with the Department of Electrical Engineering, Utsunomiya University, Japan. Since 2007,

he has been a Professor in the Graduate School of Information Science and Technology, Hokkaido University, Japan. His research interests include AC motor drive systems and static power converters.

Hideaki Arita (Member) was born in Oita, Japan, in 1977. He re-

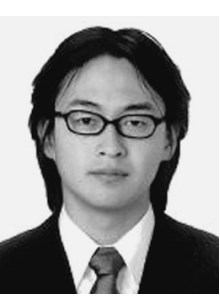
ceived B.E and M.E degrees in electrical and electronic engineering from Oita University, Japan, in 2000 and 2002, respectively. He joined the Advanced Technology R\&D Center of the Mitsubishi Electric Corporation.
Akihiro Daikoku (Member) was born in Osaka, Japan, in 1965. He

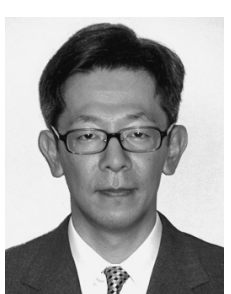
received B.E., M.E., and Ph.D. degrees in electrical engineering from Kyoto University, Japan, in 1989, 1991, and 2007, respectively. He joined the Advanced Technology R\&D Center of the Mitsubishi Electric Corporation in 1991, and since 2015 he has been a senior manager of the Electromechanical Technology Department in the corporation. Dr. Daikoku is a member of IEEE and IEEJ. 